\title{
Referred Pulpal Pain with Atypical Features in an Endodontically Treated Tooth due to Learned Phenomenon
}

\author{
${ }^{1}$ AR Vivekananda Pai, ${ }^{2}$ Mannu Vikram, ${ }^{3}$ Rachit Jain
}

\begin{abstract}
Pulpal pain does not refer to an endodontically treated tooth. Nevertheless, such a potential may exist based on a learned phenomenon due to previous treatment experiences but is rarely reported. Further, it is seldom reported to occur with atypical features. This case report is on a patient who was presented with pain in endodontically treated lower first molar (36) and recommended to undergo retreatment. However, endodontic treatment in 26 relieved the pain showing occurrence of referred pulpal pain in 36. But due to atypical referral and unknown endodontic status in 36 , only uneventful follow-up confirmed the retrospective diagnosis of referred pulpal pain in 36 . Pulpal pain can refer to an endodontically treated tooth based on learned phenomenon. Such a referral can also occur with atypical features and pose a diagnostic dilemma. Therefore, referred pulpal pain should be considered in the differential diagnosis of pain in an endodontically treated tooth.
\end{abstract}

Keywords: Diagnosis, Endodontics, Localization, Pulpitis, Referred pain.

How to cite this article: Pai ARV, Vikram M, Jain R. Referred Pulpal Pain with Atypical Features in an Endodontically Treated Tooth due to Learned Phenomenon. J Contemp Dent 2014;4(1): 60-62.

Source of support: Nil

Conflict of interest: None

\section{INTRODUCTION}

Referred pulpal pain is a type of heterotopic pain. It is said to occur due to the high degree of convergence of neurons from the pulps of mandibular teeth with those of maxillary teeth. Additionally, lack of pulpal proprioception play an important role in poor localization of pulpal pain and its referral. ${ }^{1-3}$ Typically, referred pulpal pain is characterized by an intense, boring, continuous pain and always occurs

\footnotetext{
${ }^{1}$ Professor and Head, ${ }^{2}$ Assistant Professor

${ }^{3}$ Postgraduate Student

${ }^{1,3}$ Department of Conservative Dentistry and Endodontics Manipal College of Dental Sciences, Manipal University Mangalore, Karnataka, India

${ }^{2}$ Department of Conservative Dentistry and Endodontics BP Koirala Institute of Health Sciences, Dharan, Nepal

Corresponding Author: AR Vivekananda Pai, Professor and Head, Department of Conservative Dentistry and Endodontics, Manipal College of Dental Sciences, Manipal University, Mangalore, Karnataka, India, Phone: 9880498751 e-mail: drpaivivekananda@gmail.com
}

with a previous history of mild-moderate pain in the tooth causing the pain. It tends to occur in a cephalad or upward direction and can be reproduced by manipulating the source using an intense stimulus like heat. ${ }^{1,4,5}$

Pulpal pain referral is said not to occur in a tooth which is endodontically treated or undergoing endodontic treatment. ${ }^{4}$ Nevertheless, potential for such a referral is said to exist based on a learned phenomenon due to previous treatment experiences but is rarely reported. Further, such a referral is seldom reported to occur with atypical features. This case report illustrates pulpal pain referral to an endodontically treated tooth due to learned phenomenon and its occurrence with atypical features.

\section{CASE REPORT}

A 61-year-old woman reported with pain in left mandibular first molar (36) while chewing hot and cold food items since a week. She gave a history of root canal treatment and crowns in 35 and 36. Clinical examination revealed that 35 and 36 were moderately tender on percussion with inflamed interdental papilla between them. On radiographic examination, although obturations were found satisfactory, apical radiolucency in relation to 36 and defective crown margin in 35 were detected. Due to interdental periodontitis between 35 and 36 , patient was subjected to appropriate periodontal procedures.

However, the patient complained of persistent pain in 36 and based on the clinical and radiographic examination (Fig. 1), a diagnosis of post-treatment apical periodontitis was made and retreatment was recommended in 36 .

In the subsequent visit, the intensity of pain could not be related to the objective findings observed in 36 . This led to a diagnostic uncertainty and a decision was taken to rule out any other causes for the pain in 36 .

The possibility of neuralgic pain and the pain referral from other sources could not be ascertained as the examination and medical history of the patient were not contributory.

However, a closer reinspection of the oral cavity revealed a discoloration on the approximal aspect of intact 25 and 26. Periapical radiograph showed proximal and submarginal 
deep caries in them (Fig. 2) and deep caries management was carried out.

When the patient returned with aggravated pain in 36 , she gave an intense and prolonged response to pulp tests on 26 . Therefore retreatment in 36 was postponed but a diagnosis of irreversible pulpitis followed by root canal treatment was carried out in 26 (Fig. 3).

The patient was relieved of pain in 36 following root canal treatment in 26. This showed that the pain in 36 was actually the referred pulpal pain from 26 . Uneventful outcome during 1 year follow-up confirmed the retrospective diagnosis of referred pulpal pain in 36 and ruled out the need for its retreatment (Fig. 4).

\section{DISCUSSION}

The present case report is interesting as it not only exhibited pulpal pain referral to an endodontically treated tooth based on learned phenomenon but also its occurrence with atypical features. Previous history of mild to moderate pain, which also aids in recall and pain localization, in the tooth causing pain referral is a salient feature of referred pulpal pain. ${ }^{4}$ However; this patient neither gave any history of previous pain nor located the pain in 26. It is said that the percentage of A-beta fibers, that could be sensitive to touch and pressure, is less than $5 \%$ of the myelinated nerve fibers of the pulp resulting in lack of proprioception. This lack of pulp proprioception makes it difficult for the patient to locate the actual site of pain. ${ }^{4}$ Additionally, in a pulpal inflammation, nerve impulses will originate mostly from C-fibers which have a lesser discriminatory ability leading to reduced chances of identifying the affected tooth. ${ }^{6}$

Another characteristic feature of referred pulpal pain is said to be its reproducibility by manipulating the source of pain. ${ }^{1}$ However, as 36 was endodontically treated in this patient, the above feature could not be appreciated when

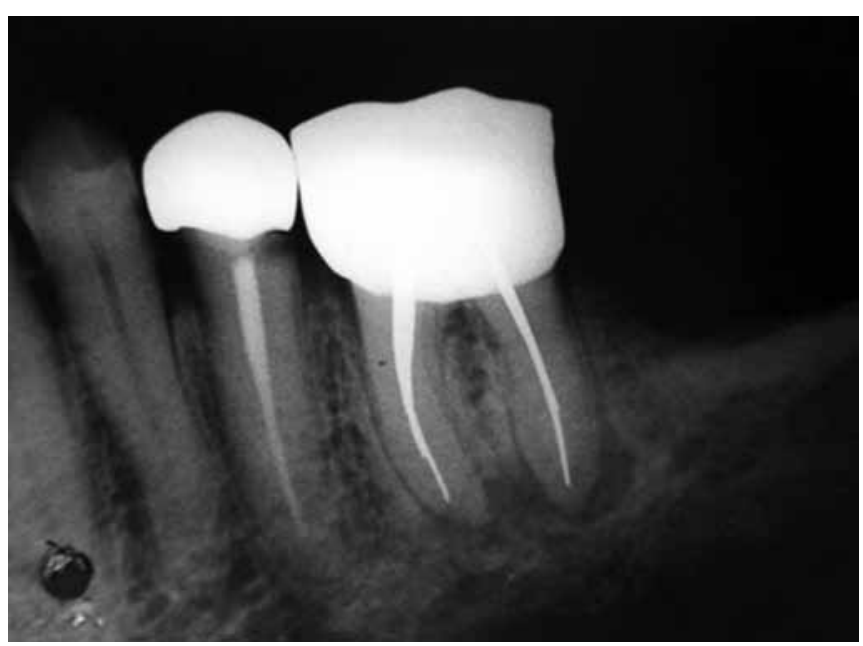

Fig. 1: Periapical radiographic view of 36 showing apical radiolucency which led to an initial diagnosis of post-treatment apical periodontitis

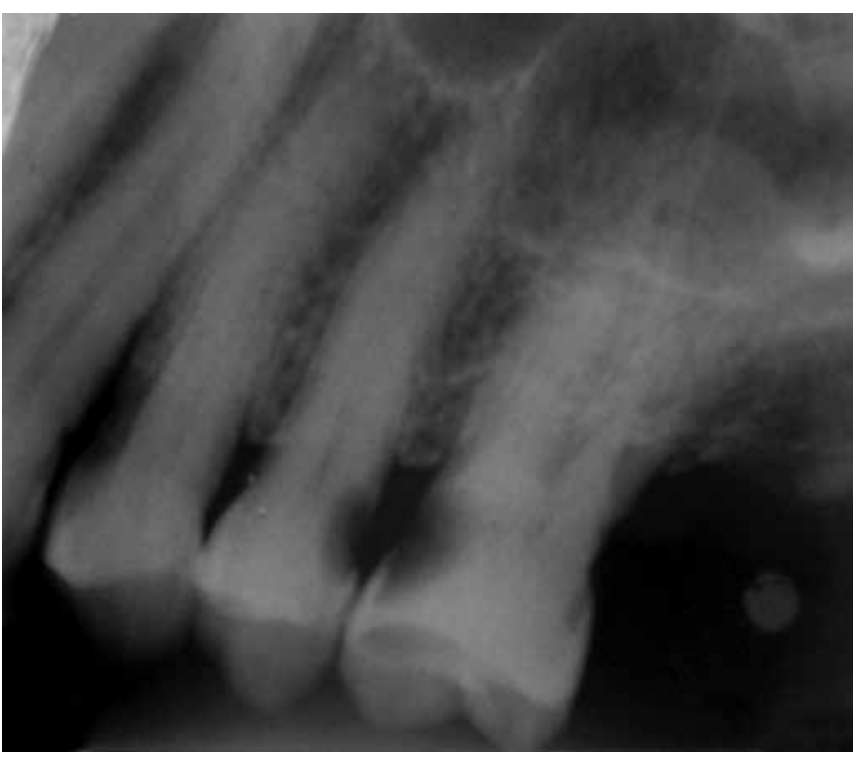

Fig. 2: Periapical radiographic view showing proximal and submarginal deep caries in 25 and 26

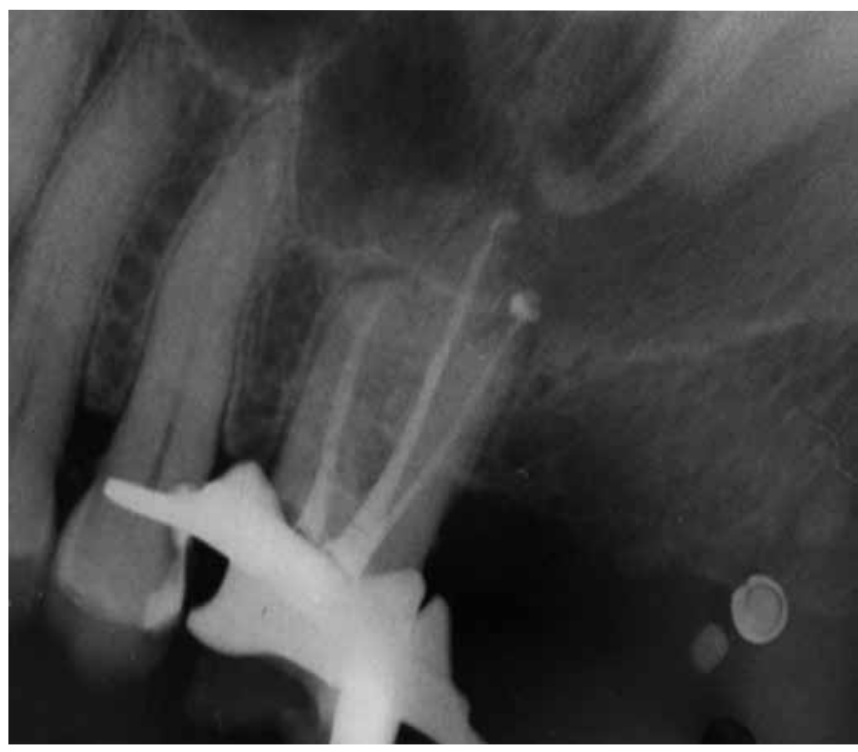

Fig. 3: Periapical radiographic view following root canal treatment in 26

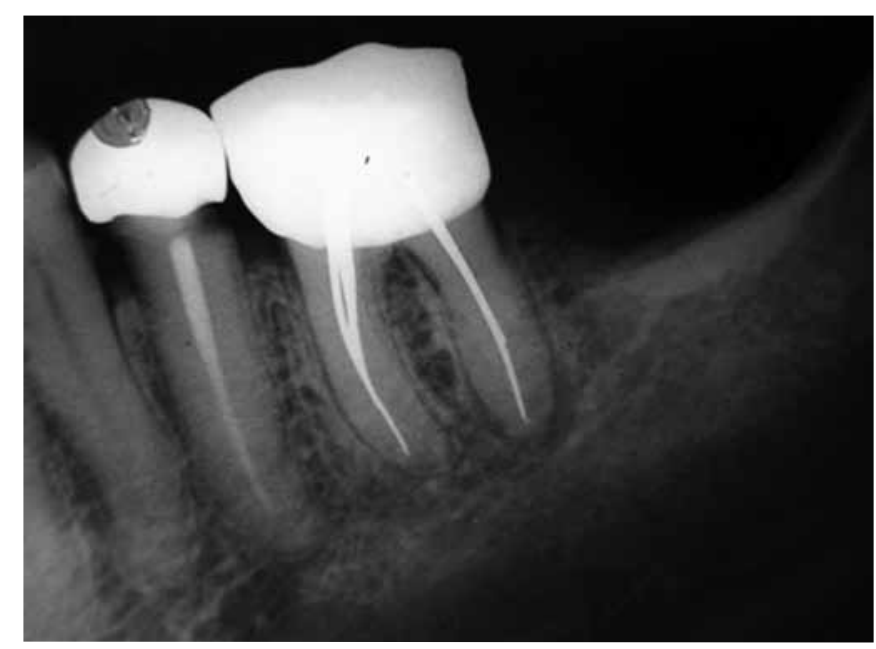

Fig. 4: One year follow-up periapical radiograph of 36 showing an uneventful outcome 
pulp testing was carried out on 26. Pulpal pain is said to refer in a cephalad or upward direction from mandibular to maxillary tooth due to laminate fashion based referral pattern. ${ }^{1}$ Contrary to this, referral in this case occurred from 26 to 36 in a downward direction.

According to projection-convergence theory, referred pulpal pain is said to occur due to high degree of convergence of neurons from the pulps of mandibular teeth with those of maxillary teeth. Hence, it is generally absent from teeth undergoing root canal treatment or root canal filled teeth. However, pain may be referred to a root canal filled tooth which may not be the actual source of the problem. ${ }^{1,3,4,7}$

Due to lack of pulpal tissue, the projection-convergence theory may not satisfactorily explain the referral of pulpal pain to an endodontically treated tooth. There is evidence that referred pain may also be a learned phenomenon. Therefore the pain may not be referred to a predicted site but to a local site of previous operation, trauma or pathosis, and this could lead to unnecessary endodontic treatment or multiple retreatments or extraction. Previously, cases of pain of muscular origin and spontaneous dissection of internal carotid artery referring to an endodontically treated tooth have been reported. ${ }^{8,9}$ However, pulpal pain referral to an endodontically treated tooth based on learned phenomenon is rarely reported.

In the present case, the poor localization of pain in 26 along with the previous experience of undergoing root canal treatment in 36 might have made the patient to learn 36, rather than 26, as the cause for pain. Further, the learned phenomenon could have been responsible for the patient to assume altered perception in 36 and respond by exhibiting tenderness to percussion in order to draw the attention toward 36. It is stated that apart from patient anxiety and stress, the anticipation of pain may lead to subjective interpretation of the applied stimuli as painful and clinical reaction to it. ${ }^{6}$

\section{CONCLUSION}

Pulpal pain can refer to an endodontically treated tooth based on learned phenomenon. Such a referral can also occur with atypical features and pose a diagnostic dilemma. Therefore, referred pulpal pain should be considered in the differential diagnosis of pain in an endodontically treated tooth.

\section{REFERENCES}

1. Mattscheck D, Law A. The nonodontogenic toothache. In: Cohen S, Hargreaves K, editors. Pathways of the pulp. 9th ed. St Louis, MO, USA: Mosby; 2006. p. 59-79.

2. Hargreaves KM. Pain mechanism of the pulpodentin complex. In: Hargreaves KM, Goodis HE, editors. Seltzer and Bender's dental pulp. Rev. 3rd ed. Hanover park: Quintessence publishing co. inc.; 2002. p. 181-203.

3. Jaeger B, Reyes MR. Nonodontogenic toothache and chronic head and neck pain. In: Ingle JI, Bakland LK, Baumgartner JC, editors. Ingle' endodontics. 6th ed. Hamilton: BC Decker inc; 2008. p. 392-467.

4. Bender IB. Pulpal pain diagnosis - a review. J Endod 2000; 26: 175-179.

5. Smulson MH, Sieraski SM. Histopathology and diseases of the dental pulp. In: Weine FS, editor. Endodontic therapy. 5th ed. St.louis, Missouri: Mosby; 1996. p. 84-165.

6. Bergeholtz G, Bindslev PH, Reit C. Textbook of endodontology. Blackwell Munksgaard; 2003.

7. Koyess E, Fares M. Referred pain: a confusing case of differential diagnosis between two teeth presenting with endo-perio problems. Int Endod J 2006;39:724-729.

8. Ernest S, Mahmoud E. Referred pain of muscular origin resembling endodontic involvement. Oral Surg Oral Med Oral Pathol Oral Radiol Endod 1991;71:223-227.

9. Roz T, Schiffman L, Schlossberg S. Spontaneous dissection of the internal carotid artery manifesting as pain in an endodontically treated molar. J Am Dent Assoc 2005;136:1556-1559. 